\title{
Generation of bright autobioluminescent bacteria by chromosomal integration of the improved lux operon ilux2
}

\author{
Carola Gregor ${ }^{1,2,3}$ \\ ${ }^{1}$ Max Planck Institute for Biophysical Chemistry, Department of NanoBiophotonics, Göttingen, Germany \\ 2Institut für Nanophotonik Göttingen e.V., Department of Optical Nanoscopy, Göttingen, Germany \\ ${ }^{3}$ Cluster of Excellence "Multiscale Bioimaging: from Molecular Machines to Networks of Excitable Cells" \\ (MBExC), University of Göttingen, Göttingen, Germany \\ Correspondence should be addressed to C.G. (carola.gregor@ifnano.de).
}

\begin{abstract}
The bacterial bioluminescence system enables light production in living cells without an external luciferin. Due to its relatively low levels of light emission, many applications of bioluminescence imaging would benefit from an increase in brightness of this system. In this report a new approach of mutagenesis and screening of the involved proteins is described that is based on the identification of mutants with improved properties under rate-limiting reaction conditions. Multiple rounds of screening in Escherichia coli resulted in the operon ilux 2 that contains 26 new mutations in the fatty acid reductase complex which provides the aldehyde substrate for the bioluminescence reaction. Chromosomal integration of ilux2 yielded an autonomously bioluminescent E. coli strain with 7-fold increased brightness compared to the previously described ilux operon. The ilux2 strain produces sufficient signal for the robust detection of individual cells and enables highly sensitive long-term imaging of bacterial propagation without a selection marker.
\end{abstract}

\section{Introduction}

Cellular light emission by the process of bioluminescence can be conveniently used to image living cells. Due to its independence from external light, bioluminescence can be easily imaged with a low-complexity optical system while background signal is virtually absent. Therefore, bioluminescence is in particular superior to fluorescence imaging in samples with high autofluorescence such as tissues. In addition, bioluminescence imaging can be used for long- 
term measurements without phototoxicity or photobleaching and also enables the observation of light-sensitive cells.

The bioluminescence system from bacteria is of particular interest since it allows imaging without external substrates. This is due to the fact that both the light-producing enzyme, the luciferase, and its substrates can be synthesized by the cell itself by simultaneous expression of the following genes: $\operatorname{lu} x A$ and $\operatorname{lu} \times B$ encoding the subunits of the luciferase, $\operatorname{lu} x C$, IuxD and $\operatorname{lu} E$ encoding the subunits of the fatty acid reductase complex and frp encoding a flavin reductase. The fatty acid reductase complex produces the aldehyde substrate for the luciferase reaction from cellular fatty acids whereas the flavin reductase generates the second substrate $\mathrm{FMNH}_{2}$. Notably, the bacterial bioluminescence system is also functional in non-bacterial cell types such as yeast and mammalian cells ${ }^{1-4}$.

One specific application of the bacterial bioluminescence system is the highly sensitive detection of bacteria in different environments. For example, it can be used to image the spreading of bacterial infections within the living host ${ }^{5-8}$ or to detect bacterial contaminations in food samples ${ }^{9-11}$. Since these measurements need to be performed over long time periods with quantifiable signal, continuous and constant expression of the involved genes must be ensured without the need for antibiotic selection. Therefore, it is desirable to integrate the genes into the bacterial chromosome rather than to express them from a plasmid which can be lost under nonselective conditions ${ }^{12-14}$. Since in the case of chromosomal expression only one copy of the transgene is present per cell, the bioluminescence emission is generally lower than from a plasmid where the copy number is usually much higher (up to 700 copies per chromosome ${ }^{15}$ ). Thus, it is particularly important to use protein variants of the bacterial bioluminescence system that produce high light levels in order to enable the reliable detection of even low cell numbers. In this paper, the generation of a new improved lux operon called ilux 2 is described that can be used for chromosomal labeling of bacteria with enhanced light emission, allowing even the detection of single E. coli cells.

\section{Results}

\section{Design of a new screening system.}

Since numerous applications of bioluminescence imaging would benefit from a genetically encodable bioluminescence system with high light output, we previously optimized the brightness obtained with the IuxCDABE operon from Photorhabdus luminescens in Escherichia coli ${ }^{16}$. We observed that bioluminescence emission in $E$. coli is not only limited by the activity of the 
luciferase itself, but also by cellular production of the substrates $\left(\mathrm{FMNH}_{2}\right.$ and fatty aldehyde). Consequently, incorporation of a flavin reductase (frp) into the lux operon and improving substrate production by several rounds of error-prone mutagenesis of luxCDE and frp substantially contributed to the increased light emission of the final improved operon ilux.

In our previous approach, we expressed all lux genes from a single vector pGEX(-) ${ }^{16}$ at relatively high levels during error-prone mutagenesis and screening. By mutagenesis of the individual genes in ilux pGEX(-), it was not possible to identify any additional mutations that further increased the bioluminescence emission. Although this could indicate that the Lux proteins are already optimized, two different explanations for this observation exist: First, supply of ATP and NADPH that are required for recycling of the substrates of the bioluminescence reaction might be limited by the cellular metabolism so that no further increase in light emission can be achieved by modifications of the Lux proteins. However, this explanation can be excluded since bioluminescence was increased by expressing ilux at higher levels from the vector pQE(-) instead of pGEX(-) ${ }^{16}$. Second, it is possible that further mutations in ilux which enhance the brightness exist, but that their effect is too small to detect them under the screening conditions used, although several such mutations combined might still be able to increase the bioluminescence emission substantially.

To explore this second possibility further, a mathematical model of the bioluminescence reaction was developed in order to determine the screening conditions under which the screening is most sensitive (i.e., even small changes in enzyme activities can be detected). Since the only observable in the screening is the bioluminescence signal rather than the enzyme activities themselves, this implies that the relative increase of the bioluminescence signal with an increase in activity of the iLux proteins should be maximized. For this purpose, the rate of light emission $B$ as a function of the substrate concentrations was first qualitatively calculated by assuming the reaction scheme in Figure $1^{17-25}$ with all rate constants set to 1 for simplicity, as explained in the Materials and Methods section ( $F: \mathrm{FMNH}_{2}, A$ : aldehyde, O: molecular oxygen, $L$ : luciferase):

$$
B=\frac{[F][A][O][L]}{(2+[A]) \cdot(1+[O]) \cdot(1+[F]+[A])}
$$

As shown in Figure 2A, the bioluminescence signal first increases with the $\mathrm{FMNH}_{2}$ and aldehyde concentrations and decreases again at high aldehyde levels as a consequence of aldehyde inhibition resulting from reversible formation of a luciferase-aldehyde complex ${ }^{22}$, with the optimal aldehyde concentration depending on the $\mathrm{FMNH}_{2}$ concentration. As can be seen from Equation 1, bioluminescence increases less than proportionally with the substrate concentrations. Therefore, an improvement in activity of the substrate-producing enzymes is not reflected to the same extent 
in the bioluminescence signal, and hence even significantly improved variants may not be detectable in the screening. Under ideal screening conditions, the relative increase in enzymatic activity would closely approach the increase in bioluminescence. To determine the optimal conditions for screening, the relative change of the bioluminescence signal with the concentrations of $\mathrm{FMNH}_{2}$ and aldehyde was therefore calculated (see Materials and Methods section). As can be seen from Figure $2 \mathrm{C}-\mathrm{E}$, the relative change in signal is highest when production of the substrate of interest is low and therefore rate-limiting for the overall bioluminescence process, whereas the other substrate is present in excess. Likewise, the luciferase is assumed to be rate-limiting for the bioluminescence reaction if high concentrations of both $\mathrm{FMNH}_{2}$ and aldehyde are present. To implement this in the new screening system, the components LuxAB, LuxCDE and Frp were analyzed separately. The component to be improved by mutagenesis was expressed at low levels from a pGEX(-) vector containing a lac promoter and medium-copy-number p15A origin of replication (ori) whereas the other components were expressed at high levels from a separate plasmid using the stronger tac promoter and high-copynumber ColE1 ori (Table 1).

\section{Generation of ilux2.}

Starting from the previously described ilux operon ${ }^{16}$, the three components iluxAB, iluxCDE and ilux frp were modified by repeated rounds of error-prone mutagenesis in independent screenings. Mutagenesis of iluxAB resulted in the new variant iluxXAB that contains 18 new mutations (Table 2) and produced fivefold higher bioluminescence signal under screening conditions than iluxAB (Figure $3 A$ ). However, when expressed at higher levels together with iluxCDEfrp from the same vector, ilux $X A B$ yielded substantially lower signal than iluxAB (Figure $3 B, C$ ) and inhibited cell growth at high expression levels for unknown reasons. ilux $X A B$ was therefore not further investigated.

Mutagenesis and screening of iluxCDE yielded the improved variant ilux2CDE containing 26 new mutations in the IuxC and IuxD genes (Table 3). Under screening conditions, ilux2CDE exhibited a 160-fold higher brightness than iluxCDE (Figure 4A). When expressed at increasing levels from the same plasmid as iluxABfrp (Figure 4B-D), the difference in brightness decreased as expected (Figure 4B,C) since LuxCDE activity became less limiting for the bioluminescence process. At very high expression levels, however, the brightness of ilux2CDE was decreased in comparison to ilux (Figure 4D). Since cell growth was concomitantly reduced, this is attributed to toxic effects resulting from excessive aldehyde production, although additionally aldehyde 
inhibition of the luciferase cannot be ruled out. The beneficial properties of ilux2CDE therefore only prevail if its expression levels are not too high.

Screening of the third component frp required special consideration. Whereas Frp activity needed to be low in comparison to LuXAB and LuxCDE for a screening that was sensitive by the above considerations, it simultaneously had to be sufficiently high to be distinguishable from the activity of other cellular flavin reductases. Using the same plasmid combinations for screening as for luxAB and luxCDE, co-expression of ilux frp did not result in higher bioluminescence than expression of iluxCDABE alone (Figure $5 \mathrm{~A}$ ). To reduce the background signal resulting from cellular flavin reductases, an $E$. coli strain was therefore engineered in which the flavin reductase gene fre was knocked out since Fre has been described to be the major FMN reductase for bioluminescence in $E$. coli ${ }^{26}$. However, bioluminescence from iluxCDABE in this strain was still not different between ilux frp pGEX(-) lac p15Aori and the empty vector (Figure 5B), indicating that the activity of other cellular flavin reductases was still too high. As a solution to this problem, ilux frp expression was increased by exchanging the lac promoter in the screening vector against the stronger tac promoter. The resulting bioluminescence could now clearly be discriminated from the empty vector (Figure 5C). Importantly, the brightness was still significantly lower than with the higher ilux frp expression from pGEX(-) Kan (Figure 5C) so that improvements in Frp could still increase the bioluminescence signal. However, it was not possible to identify brighter mutants by error-prone mutagenesis and screening, possibly because ilux frp cannot be further improved.

Overall, the brightness of ilux could only be improved by the mutations in the fatty acid reductase complex. The resulting operon was named ilux2 which consists of ilux2CDE and iluxABfrp arranged in the original order, i.e., ilux $2 C D$ iluxABEfrp.

\section{Characterization of ilux2.}

To analyze potential changes in aldehyde chain length specificity of iLux2CDE in comparison to iLuxCDE, it was attempted to purify the LuxCDE proteins to measure their activity with different fatty acids in vitro. According to our previous work ${ }^{16}$, no protein tags should be added to the LuxCDE proteins for this purpose since they may strongly reduce enzymatic activity of the fatty acid reductase complex. Unfortunately, purification of the untagged LuxCDE proteins in sufficient amounts in their active form was not successful. Therefore, the substrate specificity of iLuxCDE and iLux2CDE was compared under cellular conditions instead. For this purpose, luxCDE wt, iluxCDE and ilux2CDE were expressed from pGEX(-) Kan in E. coli and the cells were preincubated with saturated fatty acids of different chain lengths. The produced aldehyde was detected by adding a separate population of $E$. coli cells expressing iluxABfrp to the mixture. Their 
cell permeability allowed the aldehydes to diffuse from the luxCDE- into the iluxABfrp-expressing cells where they served as substrates for the bioluminescence reaction so that aldehyde production could be compared by the bioluminescence intensity.

Octanoic (C8), hexadecanoic (C16) and octadecanoic acid (C18) did not produce signal discriminable from the background signal caused by production of aldehydes from cellular substrates. For octanoic acid, this is likely due to the fact that aldehydes of eight or fewer carbon atoms are poor substrates for bacterial luciferase ${ }^{27,28}$, whereas in case of hexadecanoic and octadecanoic acid this may be due to the slow cellular entry of long-chain aldehydes ${ }^{29}$. For decanoic (C10), dodecanoic (C12) and tetradecanoic acid (C14), the bioluminescence obtained with LuxCDE wt, iLuxCDE and iLux2CDE was compared (Figure 6). Due to potential differences in cellular uptake, the signal cannot be compared between different fatty acids, but only between the LuxCDE variants. The signal from tetradecanoic acid whose corresponding aldehyde is regarded as the natural substrate for the bacterial bioluminescence reaction ${ }^{30}$ was not significantly different between LuxCDE wt, iLuxCDE and iLux2CDE. For decanoic and dodecanoic acid, iLux2CDE exhibited increased signal, whereas the brightness of iLuxCDE was surprisingly somewhat reduced compared to LuxCDE wt. It is therefore assumed that the increased bioluminescence observed with iLux2CDE is at least in part due to the enhanced utilization of cellular fatty acids other than tetradecanoic acid.

To test if iLux2CDE increases the brightness also in bacteria other than E. coli, luxCDABE wt, ilux and ilux2 were expressed from the vector pJOE7771.1 ${ }^{31}$ in Pseudomonas fluorescens (Figure 7). Both ilux and ilux2 exhibited higher brightness than IuxCDABE wt with bioluminescence emission from ilux 2 being highest, indicating that ilux 2 is also a superior reporter in bacteria other than $E$. coli.

Next, E. coli strains stably expressing the different lux operons were engineered by inserting them into the chromosome. Apart from the lux variant, the brightness also strongly depends on its expression levels, and therefore higher bioluminescence can generally be achieved with expression from a plasmid due to the higher copy number per cell. However, stable maintenance of a plasmid usually requires selection with an antibiotic resistance marker, which is not feasible or desirable for many applications such as long-term imaging of bacterial infections in living animals and plants, and hence chromosomal expression is preferable. The pGRG25 vector 32 was therefore used for site-specific chromosomal insertion of the lux operons at the attTn7 site in E. coli Top10 cells (Figure 8). In the chromosomally labeled strains, bioluminescence from ilux was only $\sim 65 \%$ higher than from luxCDABE wt. One reason for this may be that $\mathrm{FMNH}_{2}$ production by other cellular enzymes is comparatively high at the lower chromosomal expression levels, and 
therefore its supply is less rate-limiting for the bioluminescence reaction. The brightness of the ilux2 strain was $\sim 12$-fold higher than that of the luxCDABE wt strain ( 7-fold higher than ilux). Compared to the highest levels of light emission that were obtained so far with ilux expressed from the vector $\mathrm{pQE}(-)$, the stable ilux2 strain exhibits $12 \%$ of the brightness of this extrachromosomal high-copy-number system. Therefore, the ilux2 strain should enable highly sensitive and quantifiable detection of $E$. coli cells during long-term imaging without selection pressure.

\section{Bioluminescence imaging of ilux2.}

The ilux2 strain was next applied for bioluminescence imaging in different samples. First, the cell number required for detection in macroscopic samples using a commercial imaging system was determined. For this purpose, different dilutions of the stably bioluminescent $E$. coli strains were applied onto mashed potatoes and imaged with an Amersham Imager (AI) 600 RGB (Figure 9). Addition of $1 \mu \mathrm{l}$ of cell suspension resulted in spreading of the cells on the surface of the sample within an area of $\sim 5 \mathrm{~mm}$ in diameter. At the resulting cell density, around $1 \cdot 10^{4}$ and $7 \cdot 10^{3}$ cells were required to reliably detect the IuxCDABE wt and ilux strains, respectively, whereas only $1 \cdot 10^{3}$ cells were required for the ilux2 strain. In contrast, a strain chromosomally labeled with EYFP could not be detected by fluorescence with the Al 600 RGB even at the highest cell number of $10^{6}$ cells used. While bioluminescence imaging is limited by the low number of emitted photons, fluorescence imaging of samples with high autofluorescence such as food products or living tissues mostly suffers from the high fluorescence background. Consequently, discrimination of the fluorescence signal of the EYFP strain was impossible despite the higher brightness of the image (Figure 9D), demonstrating the superiority of bioluminescence imaging in these samples.

Imaging with the Al $600 \mathrm{RGB}$ was performed at room temperature instead of $37^{\circ} \mathrm{C}$, where bioluminescence levels are 2-3 times higher ${ }^{16}$. In addition, a relatively large distance between sample and objective lens was used to record a large field of view. Therefore, even lower cell numbers should be detectable under different imaging conditions. To test if even single cells can be detected, the luxCDABE wt, ilux and ilux2 strains were imaged with a custom-built microscope as described in ${ }^{4,16,33}$. As can be seen in Figure 10, only the ilux 2 strain could be imaged with a high signal-to-noise ratio, demonstrating the usefulness of this strain also for single-cell applications.

Finally, the ilux2 strain was applied for long-term imaging of growth of $E$. coli cells in different food samples (Figure 11, Movies 1-5). Since only viable cells emit bioluminescence, both growth and spreading of the cells in the sample can be monitored over time. Cells grown on slices of raw 
cucumber and potato (Figure 11A,B, Movies 1 and 2) reached maximum bioluminescence after around 5 and 8 hours, respectively. Interestingly, the signal was not homogeneously distributed over the inoculated region, but especially on potato numerous bright spots appeared that changed their position over time. It is speculated that these bright spots emerge in regions of elevated supply with nutrient such as starch which might originate from breakdown of the potato cells. On mashed potatoes and egg yolk, the bioluminescence signal in the inoculated region was more uniform (Figure 11C,D, Movies 3 and 4). The signal strongly increased over time and reached its maximum after more than $24 \mathrm{~h}$, indicating a high nutrient availability that supports long-term growth at high cell numbers. Since movement of the cells on the semi-solid surfaces was very limited, the signal finally started to decay at the center of the inoculated region where nutrients were depleted first whereas at the edges high bioluminescence was sustained for longer times. For comparison, Figure 11E and Movie 5 show the bioluminescence from milk that was chosen as a liquid sample. Although the sample was not moved after inoculation, the cells quickly spread over the complete dish, but were not evenly distributed in the sample during the entire $2 \mathrm{~d}$ observation time. Maximum bioluminescence was observed after $\sim 11 \mathrm{~h}$ and at the end of the $48 \mathrm{~h}$ observation time bioluminescence was barely detectable, presumably due to the depletion of nutrients and a drop in $\mathrm{pH}$ value. Together, these results demonstrate the utility of ilux2 as a highly sensitive reporter for long-term imaging of bacteria in samples with high autofluorescence at an excellent signal-to-noise ratio.

\section{Discussion}

By optimization of the screening conditions, it was demonstrated that light emission from the bacterial bioluminescence system can be improved under certain expression conditions compared to the previously engineered ilux system ${ }^{16}$. This was achieved by component-wise mutagenesis of the involved proteins and screening under rate-limiting reaction conditions so that smaller improvements became detectable, which added up to a significant enhancement in light emission after multiple screening steps. It should be noted that no absolute value for the improvement in brightness can be specified since it strongly depends on the expression levels of all involved proteins. In addition, the overall process of bioluminescence is affected by the cellular availability of the substrates (e.g., fatty acids), resulting in a complex relationship between lux expression and bioluminescence emission. Moreover, toxic effects due to accumulation of the reaction products such as aldehydes can lead to adverse effects and reduce cell growth at elevated expression levels, which in turn decreases light emission. Therefore, bioluminescence 
from the newly generated ilux2 operon is only increased at moderate expression levels. Nevertheless, the described screening system enables the generation of improved protein variants that would not be accessible otherwise. The presented screening strategy may therefore also be useful for the optimization of other processes involving multiple enzymes.

The enhanced light emission of the ilux2 system results from 26 mutations in the fatty acid reductase complex (15 mutations in iluxC, 11 mutations in iluxD). The results demonstrate that the increased light output is at least in part due to the improved utilization of fatty acids other than tetradecanoic acid for which the fatty acid reductase complex has the highest activity ${ }^{34,35}$. A broadened substrate spectrum of iLux2CDE relative to LuxCDE wt would be in line with the high specificity of the wild-type fatty acid reductase complex for tetradecanoic acid since fatty acids that are potentially more abundant in the cell could also be used for the process of bioluminescence. The luciferase itself discriminates less between different chain lengths of its aldehyde substrate ${ }^{27,28}$ so that elevated production of aldehydes other than tetradecanoic aldehyde is expected to increase light emission. Besides the saturated fatty acids that were investigated in this study, also other substrates may be used with different specificity in the cell, such as unsaturated fatty acids or acyl-ACP and acyl-CoA esters.

The increased brightness of ilux2 is particularly useful for the generation of stably autobioluminescent bacteria by chromosomal integration of the operon. These stable strains could for instance be used to image the spreading of bacterial infections in living animals and plants with enhanced sensitivity. Since bioluminescence emission requires metabolic activity, only living bacteria emit light. Therefore, bioluminescence imaging does not only yield information about the localization of the bacteria within the host, but also about their viability so that processes such as bacterial elimination by drugs or the immune system can be observed. In addition, ilux2labeled strains can be used for in vitro applications, e.g. for drug screening to develop new antibacterial agents or to study antibiotic resistances. Another important application is the visualization of pathogenic bacteria on food samples to contribute to food safety. For example, bacterial growth can be studied under different storage conditions over long periods of time and different methods for preserving foodstuffs can be tested. It is therefore expected that ilux 2 will be a valuable reporter for highly sensitive bacterial imaging in samples that are not accessible by fluorescence measurements. 


\section{Materials and Methods}

\section{Calculation of optimal screening conditions.}

To determine the optimal conditions for the screening, a mathematical model was set up based on the reaction scheme in Figure 1. For better clarity, the following abbreviations are used below: A: aldehyde (RCHO), F: FMNH 2 , O: $\mathrm{O}_{2}$, L: LuxAB, LA: LuxAB·RCHO, LF: LuxAB·FMNH 2 , LFO: LuXAB·FMNHOOH, LFOA: LuXAB·FMNHOOR. It was first aimed at including also reactions for the production of aldehyde from fatty acids by LuxCDE and of $\mathrm{FMNH}_{2}$ from $\mathrm{FMN}$ by Frp in order to express the rate of light emission as a function of the reaction rate constants and concentrations of LuxAB, LuxCDE and Frp. However, since the resulting system was too complex to utilize its analytical solution, the concentrations of $\mathrm{FMNH}_{2}$ and aldehyde were used as variables instead of LuxCDE and Frp activity.

Based on the reaction scheme, the emitted bioluminescence $B$ is proportional to the concentration of the LFOA complex:

$$
B=k_{9}[L F O A]
$$

Under cellular steady-state conditions, the concentrations of LA, LF, LFO and LFOA remain constant. Therefore, the following rate equations can be deduced from Figure 1:

$$
\begin{gathered}
\frac{d[L A]}{d t}=k_{1}[L][A]-k_{2}[L A]=0 \\
\frac{d[L F]}{d t}=k_{3}[L][F]-k_{4}[L F]-k_{5}[O][L F]=0 \\
\frac{d[L F O]}{d t}=k_{5}[O][L F]-k_{6}[L F O]-k_{7}[A][L F O]+k_{8}[L F O A]=0 \\
\frac{d[L F O A]}{d t}=k_{7}[A][L F O]-k_{8}[L F O A]-k_{9}[L F O A]=0
\end{gathered}
$$

In addition, the concentrations of all intermediates must equal the total cellular concentration of luciferase $[L]_{0}$ (for simplicity, other existing states of the luciferase are neglected since their concentration is also constant under steady-state conditions):

$$
[L]_{0}=[L]+[L A]+[L F]+[L F O]+[L F O A]
$$

This set of six equations was solved with a custom-written Matlab (The MathWorks, Inc., Natick, Massachusetts) script. Since the rate constants $k_{i}$ for the utilized proteins are unknown, they were 
all set to 1 for a qualitative description of the process, yielding the following equation for the bioluminescence emission:

$$
B=\frac{[F][A][O][L]_{0}}{(2+[A]) \cdot(1+[O]) \cdot(1+[F]+[A])}
$$

From this equation, it is evident that the bioluminescence signal increases less than proportionally with the substrate concentrations $[F]$ and $[A]$, i.e., an increase in $[F]$ or $[A]$ results in a smaller percentage change of $\mathrm{B}$. For optimal sensitivity in the screening, the relative signal change with varying concentrations of the reactants $(F, A$ and $L$, respectively) must be maximal. This was calculated as the derivative of $B$ with respect to the screened reactant and normalized to $B$, yielding for $\mathrm{F}$ :

$$
\begin{gathered}
\frac{d B}{d[F]}=\frac{[A][O][L]_{0} \cdot(1+[A])}{(2+[A]) \cdot(1+[O]) \cdot(1+[F]+[A])^{2}} \\
\frac{d B / d[F]}{B}=\frac{1+[A]}{[F] \cdot(1+[F]+[A])}
\end{gathered}
$$

and for $A$ :

$$
\begin{gathered}
\frac{d B}{d[A]}=\frac{[F][O][L]_{0} \cdot\left(2+2[F]-[A]^{2}\right)}{(2+[A])^{2} \cdot(1+[O]) \cdot(1+[F]+[A])^{2}} \\
\frac{d B / d[A]}{B}=\frac{2+2[F]-[A]^{2}}{[A] \cdot(2+[A]) \cdot(1+[F]+[A])}
\end{gathered}
$$

These results are plotted in Fig. 2 with [O] and [L]o set to 1.

\section{Construction of screening vectors.}

The vector pGEX(-) for tagless expression of the Lux proteins was generated as described previously ${ }^{16}$. For the two-plasmid based screening, another expression vector pGEX(-) Kan was generated in which the ampicillin resistance gene was exchanged against a kanamycin resistance marker in the following way: the kanamycin resistance gene was amplified by PCR using the primers KanR Spel fwd and KanR Ascl rev. The vector backbone was amplified with the primers pGEX(-) Spel rev and pGEX(-) Ascl fwd. Both parts were digested with Spel and Ascl and subsequently ligated. 
For low-level expression, the tac promoter in pGEX(-) was exchanged against a lac promoter by PCR of the vector with the primers pGEX(-) +lac BamHI rev and pGEX(-) BamHI fwd and digestion with BamHI, followed by ligation without an insert.

To maintain two plasmids in the same cell during the screening, the ColE1 origin of replication in pGEX(-) was exchanged against the p15A origin of replication (p15Aori). For this purpose, the p15A origin of replication was PCR amplified with the primers p15Aori Bglll fwd and p15Aori Avrll rev, whereas the vector backbone was amplified with the primers pGEX(-) Bglll rev and pGEX(-) Avrll fwd. Both parts were digested with Bglll and Avrll and subsequently ligated.

\section{Error-prone mutagenesis and screening.}

For mutagenesis of luxAB, iluxAB was amplified from ilux pGEX(-) described in ${ }^{16}$ and cloned into the vector pGEX(-) lac p15Aori between the BamHI and Notl restriction sites. The remaining genes iluxCDEfrp were cloned into pGEX(-) Kan for high-level expression between the BamHI and Notl restriction sites. Error-prone mutagenesis of iluxAB in pGEX(-) lac p15Aori was performed as described previously ${ }^{16}$. For screening, the ligation mixture was transformed into electrocompetent $E$. coli Top10 cells already containing the plasmid iluxCDEfrp pGEX(-) Kan. The cells were then spread out on LB agar plates containing ampicillin and kanamycin (both $50 \mu \mathrm{g} / \mathrm{ml}$ ) and incubated overnight at $37^{\circ} \mathrm{C}$. Expression was not induced with IPTG. The following day, the plates were imaged with an Amersham Imager 600 RGB (AI 600 RGB, GE Healthcare, Chicago, Illinois) and the clones with the highest bioluminescence signal were selected and used for the next round of mutagenesis. Screening and mutagenesis of luxCDE were performed in analogy to IUXAB using the plasmids iluxCDE pGEX(-) lac p15Aori and iluxABfrp pGEX(-) Kan.

For mutagenesis of frp, a Top10 fre knock-out (freKO) strain was generated in order to reduce $\mathrm{FMNH}_{2}$ production by the endogenous flavin reductase encoded by the fre gene. The knock-out strain was constructed by CRISPR using the system described in ${ }^{36}$ with the target sequence GCGGCCTTTTCTTTTCGTGC and the oligonucleotide $\mathrm{T}^{*} \mathrm{C}^{*} \mathrm{~A}^{*} \mathrm{~T}{ }^{*}$ CCATCACTACCATCAAATACTGAGCGGCTCAAAAAGAAAAGGCCGCGTCTGGCA CGATGCGGACACGATATACGGT for recombineering ( ${ }^{*}$ indicates phosphorothioate bonds).

\section{Comparison of LuxCDE wt, iLuxCDE and iLux2CDE activity with different fatty acids.}

luxCDE wt, iluxCDE, ilux2CDE and iluxABfrp were expressed in Top10 cells from the vector pGEX(-) Kan. For each construct, four colonies were spread out onto LB agar plates containing $50 \mu \mathrm{g} / \mathrm{ml}$ kanamycin and $20 \mu \mathrm{M}$ IPTG and incubated overnight at $37^{\circ} \mathrm{C}$. The following day, cells were resuspended in LB medium and the $\mathrm{OD}_{600}$ was adjusted 0.5 (luxCDE wt, iluxCDE, ilux2CDE) 
or 1.0 (iluxABfrp). $100 \mu \mathrm{l}$ of the luxCDE cell suspension was mixed with $3 \mu \mathrm{l}$ of fatty acid solution (octanoic, decanoic, dodecanoic, tetradecanoic, hexadecanoic or octadecanoic acid, all dissolved in ethanol at a concentration of $10 \mathrm{mM}$ ) and incubated for $5 \mathrm{~min}$ at room temperature. Next, $10 \mu \mathrm{l}$ of the luxABfrp cell suspension were added and incubated for $5 \mathrm{~min}$ at room temperature before starting the measurement. Bioluminescence intensity was determined with the Al $600 \mathrm{RGB}$. The backgound signal from a sample without addition of fatty acid was subtracted.

\section{Lux expression in Pseudomonas fluorescens}

Pseudomonas fluorescens (DSM 50090) was obtained from DSMZ (Leibniz Institute DSMZ German Collection of Microorganisms and Cell Cultures $\mathrm{GmbH}$, Braunschweig, Germany) and cultivated in LB medium at $30^{\circ} \mathrm{C}$. For expression in P. fluorescens, luxCDABE wt, ilux and ilux2 were inserted into the expression vector pJOE7771.1 ${ }^{31}$ between the BamHI and Spel restriction site. Plasmid transformation was performed by electroporation in $2 \mathrm{~mm}$ cuvettes at $2.5 \mathrm{kV}$ using a capacitance of $25 \mu \mathrm{F}$ and a resistance of $200 \Omega$. Cells were grown at $30^{\circ} \mathrm{C}$ on LB agar plates containing $50 \mu \mathrm{g} / \mathrm{ml}$ kanamycin.

\section{Generation of stably bioluminescent $E$. coli strains.}

For chromosomal insertion, luxCDABE wt, ilux, ilux2 and EYFP were first cloned into a pGEX(-) vector containing an Ascl restriction site in front of the tac promoter. This vector was generated by PCR of pGEX(-) with the primers pGEX(-) Ascl tac fwd and pGEX(-) Ascl tac rev, followed by digestion of the PCR product with Ascl and ligation without an insert. In the next step, IUxCDABE $w t$, ilux, ilux2 and EYFP were cut from previously generated pGEX(-) plasmids with BamHI and Notl and ligated into the new vector pGEX(-) Ascl tac digested with the same enzymes. Finally, the complete inserts including the tac promoter were cut out from the newly generated plasmids using Ascl and Notl and ligated into the vector pGRG25 described in ${ }^{32}$ that was digested with the same enzymes.

Insertion of the transgenes from pGRG25 into E. coli Top10 was performed as described in ${ }^{32}$. Curing of the pGRG25 plasmid was checked by ampicillin sensitivity. Correct insertion of the transgenes into the chromosome was verified by colony PCR of the final strains using the primers attTn7 fwd and luxC EcoRI rev for luxCDABE wt, ilux and ilux2, frp Sall fwd and attTn7 rev for ilux and ilux2 and attTn7 fwd and attTn7 rev for EYFP. 


\section{Bioluminescence imaging.}

To compare the brightness of different variants, bacteria were transformed with the indicated plasmids and grown on LB agar plates containing the appropriate antibiotics overnight. The following day, single colonies were spread out onto a new LB agar plate. If not stated otherwise, plates were incubated at $37^{\circ} \mathrm{C}$ overnight. The following day, plates were imaged with the $\mathrm{Al}$ 600 RGB to quantify the bioluminescence signal.

The number of detectable cells of stable Top10 strains containing luxCDABE wt, ilux, ilux2 and EYFP on mashed potatoes was compared in 24-well plates. Each well was filled with freshly prepared mashed potatoes (consisting of boiled potatoes and water only) up to a height of around $0.5 \mathrm{~cm}$. Cells grown at $37^{\circ} \mathrm{C}$ on LB agar plates without antibiotics or IPTG were resuspended in PBS and $1 \mu$ of different dilutions of the cell suspension was added at the center of each well. Plates were imaged at room temperature with the Al 600 RGB. For the luxCDABE wt, ilux and ilux2 strains, bioluminescence was recorded for $10 \mathrm{~min}$. For the EYFP strain, fluorescence was recorded with an excitation wavelength of $520 \mathrm{~nm}$, a 605BP40 filter and a 1-s exposure time. The number of viable cells was determined by plating different dilutions of the cell suspensions on LB agar plates and counting the number of colony-forming units (cfu) after incubation overnight at $37^{\circ} \mathrm{C}$.

Imaging of single $E$. coli cells was performed using a custom-built microscope as described previously ${ }^{4,16,33}$. Briefly, cells grown on LB agar plates were resuspended in LB medium and imaged between a coverslip and LB agar pad. Imaging was performed in a stage top incubation system (Okolab, Ottaviano, Italy) at $37^{\circ} \mathrm{C}$. A $60 \times$ oil immersion objective lens (PlanApo $\mathrm{N}$ 60x/1.42, Olympus, Shinjuku, Tokyo, Japan) and an electron-multiplying charge-coupled device (EMCCD) camera (iXon EMCCD DU-897E-CS0-\#BV, Andor Technology Ltd, Belfast, Northern Ireland) with the camera sensor cooled to $-100{ }^{\circ} \mathrm{C}$ were used to detect the bioluminescence light. To select and focus the cells, cells were transformed with EYFP pGEX(-) beforehand. EYFP fluorescence was excited with a laser (Cobolt Calypso $50 \mathrm{~mW}$, Hübner Photonics, Kassel, Germany) at $491 \mathrm{~nm}$. Bright pixels were filtered out using a custom-written Matlab script as described in 4, 16, 33 .

Movies of the stable ilux2 Top10 strain on food products were taken with the AI 600 RGB. Slices of raw cucumber and potato, raw egg yolk, mashed potatoes (consisting of boiled potatoes and water only) and UHT milk were placed in transparent 6-cm dishes, inoculated with around $5 \cdot 10^{6} \mathrm{cfu}$ in PBS and covered with a lid to prevent dehydration of the samples. One image (exposure time $1 \mathrm{~min}$ or $5 \mathrm{~s}$ ) was recorded per hour for $48 \mathrm{~h}$. 


\section{Acknowledgements}

I wish to thank Stefan W. Hell for providing access to the optical setup for single-cell imaging and Steffen J. Sahl for feedback on the manuscript. pKDsgRNA-ack, pCas9-CR4 and pKDsgRNAp15 were a gift from Kristala Prather (Addgene plasmids \#62654-62656). pGRG25 was a gift from Nancy Craig (Addgene plasmid \#16665). pJOE7771.1 was a gift from Josef Altenbuchner (Addgene plasmid \#135077).

\section{Competing Interests}

The Max Planck Society filed a patent application (EP21209450.2) related to the use of ilux2.

\section{References}

1. R. K. Gupta, S. S. Patterson, S. Ripp, M. L. Simpson, G. S. Sayler, Expression of the Photorhabdus luminescens lux genes (IuxA, B, C, D, and E) in Saccharomyces cerevisiae. FEMS Yeast Res 4, 305-313 (2003).

2. D. M. Close et al., Autonomous bioluminescent expression of the bacterial luciferase gene cassette (lux) in a mammalian cell line. PLOS One 5, e12441 (2010).

3. T. Xu, S. Ripp, G. S. Sayler, D. M. Close, Expression of a humanized viral 2A-mediated lux operon efficiently generates autonomous bioluminescence in human cells. PLOS One 9, e96347 (2014).

4. C. Gregor et al., Autonomous bioluminescence imaging of single mammalian cells with the bacterial bioluminescence system. Proc Natl Acad Sci USA 116, 26491-26496 (2019).

5. N. A. Kuklin et al., Real-time monitoring of bacterial infection in vivo: development of bioluminescent staphylococcal foreign-body and deep-thigh-wound mouse infection models. Antimicrob Agents Chemother 47, 2740-2748 (2003).

6. S. Massey et al., In vivo bioluminescence imaging of Burkholderia mallei respiratory infection and treatment in the mouse model. Front Microbiol 2, 174 (2011).

7. J. Du et al., Surfactant protein $D$ attenuates acute lung and kidney injuries in pneumonia-induced sepsis through modulating apoptosis, inflammation and NF-kB signaling. Sci Rep 8, 15393 (2018).

8. K. Briestenská, M. Mikušová, K. Tomčíková, F. Kostolanský, E. Varečková, Quantification of bacteria by in vivo bioluminescence imaging in comparison with standard spread plate method and 
reverse transcription quantitative PCR (RT-qPCR). Arch Microbiol 10.1007/s00203-021-02458-5 (2021).

9. J. M. Baker, M. W. Griffiths, D. L. Collins-Thompson, Bacterial bioluminescence: Applications in food microbiology. J Food Prot 55, 62-70 (1992).

10. A. Karsi et al., Development of bioluminescent Salmonella strains for use in food safety. BMC Microbiol 8, 10 (2008).

11. R. Morrissey, C. Hill, M. Begley, Shining light on food microbiology; applications of Lux-tagged microorganisms in the food industry. Trends Food Sci Technol 32, 4-15 (2013).

12. Y. Inoue, Spontaneous loss of antibiotic-resistant plasmids transferred to Escherichia coli in experimental chronic bladder infection. Int J Urol 4, 285-288 (1997).

13. M. A. Smith, M. J. Bidochka, Bacterial fitness and plasmid loss: the importance of culture conditions and plasmid size. Can J Microbiol 44, 351-355 (1998).

14. L. De Gelder, J. M. Ponciano, P. Joyce, E. M. Top, Stability of a promiscuous plasmid in different hosts: no guarantee for a long-term relationship. Microbiology 153, $452-463$ (2007).

15. M. P. Mayer, A new set of useful cloning and expression vectors derived from pBlueScript. Gene 163, 41-46 (1995).

16. C. Gregor, K. C. Gwosch, S. J. Sahl, S. W. Hell, Strongly enhanced bacterial bioluminescence with the ilux operon for single-cell imaging. Proc Natl Acad Sci USA 115, 962-967 (2018).

17. A. Eberhard, J. W. Hastings, A postulated mechanism for the bioluminescent oxidation of reduced flavin mononucleotide. Biochem Biophys Res Commun 47, 348-353 (1972).

18. J. W. Hastings, C. Balny, C. L. Peuch, P. Douzou, Spectral properties of an oxygenated luciferaseflavin intermediate isolated by low-temperature chromatography. Proc Natl Acad Sci USA 70, 34683472 (1973).

19. J. W. Hastings, C. Balny, The oxygenated bacterial luciferase-flavin intermediate. Reaction products via the light and dark pathways. J Biol Chem 250, 7288-7293 (1975).

20. M. Kurfürst, S. Ghisla, J. W. Hastings, Characterization and postulated structure of the primary emitter in the bacterial luciferase reaction. Proc Natl Acad Sci USA 81, 2990-2994 (1984).

21. P. Macheroux, S. Ghisla, J. W. Hastings, Spectral detection of an intermediate preceding the excited state in the bacterial luciferase reaction. Biochemistry 32, 14183-14186 (1993).

22. H. M. Abu-Soud, A. C. Clark, W. A. Francisco, T. O. Baldwin, F. M. Raushel, Kinetic destabilization of the hydroperoxy flavin intermediate by site-directed modification of the reactive thiol in bacterial luciferase. J Biol Chem 268, 7699-7706 (1993). 
23. W. A. Francisco et al., Deuterium kinetic isotope effects and the mechanism of the bacterial luciferase reaction. Biochemistry 37, 2596-2606 (1998).

24. B. Lei, Q. Ding, S.-C. Tu, Identity of the emitter in the bacterial luciferase luminescence reaction: Binding and fluorescence quntum yield studies of 5-decyl-4a-hydroxy-4a,5-dihydroriboflavin-5'phosphate as a model. Biochemistry 43, 15975-15982 (2004).

25. R. Tinikul, P. Chaiyen, "Structure, mechanism, and mutation of bacterial luciferase" in Bioluminescence: Fundamentals and Applications in Biotechnology - Volume 3, G. Thound, R. Marks, Eds. (Springer, Berlin, Heidelberg, 2014), vol. 154, pp. 47-74.

26. Z. T. Campbell, T. O. Baldwin, Fre is the major flavin reductase supporting bioluminescence from Vibrio harveyi luciferase in Escherichia coli. J Biol Chem 284, 8322-8328 (2009).

27. J. W. Hastings, J. Spudich, G. Malnic, The influence of aldehyde chain length upon the relative quantum yield of the bioluminescent reaction of Achromobacter fischeri. $J$ Biol Chem 238, 31003105 (1963).

28. J. W. Hastings et al., Structurally distinct bacterial luciferases. Biochemistry 8, 4681-4689 (1969).

29. E. Meighen, Molecular biology of bacterial bioluminescence. Microbiol Rev 55, 123-142 (1991).

30. S. Ulitzur, J. W. Hastings, Evidence for tetradecanal as the natural aldehyde in bacterial bioluminescence. Proc Natl Acad Sci USA 76, 265-267 (1979).

31. J. Hoffmann, J. Altenbuchner, Functional characterization of the mannitol promoter of Pseudomonas fluorescens DSM 50106 and its application for a mannitol-inducible expression system for Pseudomonas putida KT2440. PLOS One 10, e0133248 (2015).

32. G. J. McKenzie, N. L. Craig, Fast, easy and efficient: site-specific insertion of transgenes into Enterobacterial chromosomes using Tn7 without need for selection of the insertion event. BMC Microbiol 6, 39 (2006).

33. C. Gregor, Bioluminescent imaging of single bacterial cells using an enhanced ilux operon. Methods Mol Biol 2081, 43-52 (2020).

34. S. Ulitzur, J. W. Hastings, Myristic acid stimulation of bacterial bioluminescence in "aldehyde" mutants. Proc Natl Acad Sci USA 75, 266-269 (1978).

35. D. Riendeau, E. Meighen, Evidence for a fatty acid reductase catalyzing the synthesis of aldehydes for the bacterial bioluminescent reaction. Resolution from luciferase and dependence on fatty acids. J Biol Chem 254, 7488-7490 (1979).

36. C. R. Reisch, K. L. J. Prather, The no-SCAR (Scarless Cas9 Assisted Recombineering) system for genome editing in Escherichia coli. Sci Rep 5, 15096 (2015). 


\section{Tables}

Table 1. Plasmid combinations used for error-prone mutagenesis. pGEX(-) was used as the vector backbone.

\begin{tabular}{|l|l|l|l|l|l|l|l|}
\hline \multicolumn{3}{|l|}{ Plasmid for error-prone mutagenesis } & \multicolumn{3}{l|}{ Plasmid containing the remaining lux genes } \\
\hline Insert & Promoter & $\begin{array}{l}\text { Origin of } \\
\text { replication }\end{array}$ & Resistance & Insert & Promoter & $\begin{array}{l}\text { Origin of } \\
\text { replication }\end{array}$ & Resistance \\
\hline iluxAB & lac & p15Aori & ampicillin & iluxCDEfrp & tac & ColE1 & kanamycin \\
\hline iluxCDE & lac & p15Aori & ampicillin & iluxABfrp & tac & ColE1 & kanamycin \\
\hline ilux frp & tac & p15Aori & ampicillin & iluxCDABE & tac & ColE1 & kanamycin \\
\hline
\end{tabular}

Table 2. Mutations identified during the screening of $I u x A B$ in addition to the mutations in ilux.

\begin{tabular}{|l|l|}
\hline Gene & Mutations \\
\hline iluxXA & L24M, I76V, Q86H, M139L, K283R, N289S, I298T, Y360- \\
\hline iluxXB & T28S, Q95H, D112G, K193R, K238N, Y276F, M306T, V311A, D317A, H323L \\
\hline
\end{tabular}

Table 3. Mutations contained in ilux2 in addition to the mutations in ilux.

\begin{tabular}{|l|l|}
\hline Gene & Mutations \\
\hline ilux2C & $\begin{array}{l}\text { T10N, N27T, N45T, I47T, E54D, D74N, S77P, L132I, D135G, T216I, Y248N, } \\
\text { K345N, V348A, K414E, T445K }\end{array}$ \\
\hline ilux2D & N3K, I90T, K106N, N108S, M112V, N165K, D168V, K234R, V251A, D274N, E300V \\
\hline
\end{tabular}

Table 4. Primer sequences.

\begin{tabular}{|l|l|}
\hline Name & Sequence $\left(5^{\prime} \rightarrow 3^{\prime}\right)$ \\
\hline KanR Spel fwd & GTTGATACTAGTATGAGCCATATTCAACGG \\
\hline KanR Ascl rev & GTTGATGGCGCGCCTTAGAAAAACTCATCGAG \\
\hline pGEX(-) Spel rev & GTTGCAACTAGTACTCTTCCTTTTTCAATATTATTG \\
\hline pGEX(-) Ascl fwd & GTTGATGGCGCGCCCTGTCAGACCAAGTTTACTCA \\
\hline pGEX(-) +lac BamHI rev & $\begin{array}{l}\text { TCCGGGGATCCTGTTTCCTGTGTGAAATTGTTATCCGCTCA } \\
\text { CAATTCCACACCAACATACGAGCCGGAAGCATAAAGTGTAA } \\
\text { ACAGCTCATTTCAGAAT }\end{array}$ \\
\hline pGEX(-) BamHl fwd & GGATAACAATTTCACACAGGAAACAGGATCC \\
\hline p15Aori Bglll fwd & ATCTTCAGATCTTTGAGATCGTTTTGGTCT \\
\hline p15Aori Avrll rev & TTGATCCCTAGGTTTCCATAGGCTCCGCCC \\
\hline pGEX(-) Bglll rev & AACTAGAGATCTGAAGATCCTTTGATCTTTTCTAC \\
\hline pGEX(-) Avrll fwd & GTTGCACCTAGGAACGCCAGCAACGCGGCC \\
\hline pGEX(-) Ascl tac fwd & TTGATAGGCGCGCCTTGACAATTAATCATCGGCTCGTATAA \\
\hline pGEX(-) Ascl tac rev & GTTACTGGCGCGCCCAGCTCATTTCAGAATATTTGCCAG \\
\hline attTn7 fwd & GATGCTGGTGGCGAAGCTGT \\
\hline luxC EcoRI rev & $\begin{array}{l}\text { GTTGATGAATTCACTTTTTACCTATTATGGGACAAATACAAG } \\
\text { GAAC }\end{array}$ \\
\hline
\end{tabular}


bioRxiv preprint doi: https://doi.org/10.1101/2021.12.10.472100; this version posted December 11, 2021. The copyright holder for this preprint (which was not certified by peer review) is the author/funder. All rights reserved. No reuse allowed without permission.

\begin{tabular}{|l|l|}
\hline frp Sall fwd & $\begin{array}{l}\text { GTAACTGTCGACCTAAGGAGAAAGAAATGGTGAAGATACA } \\
\text { G }\end{array}$ \\
\hline attTn7 rev & GATGACGGTTTGTCACATGGA \\
\hline
\end{tabular}




\section{Figures}

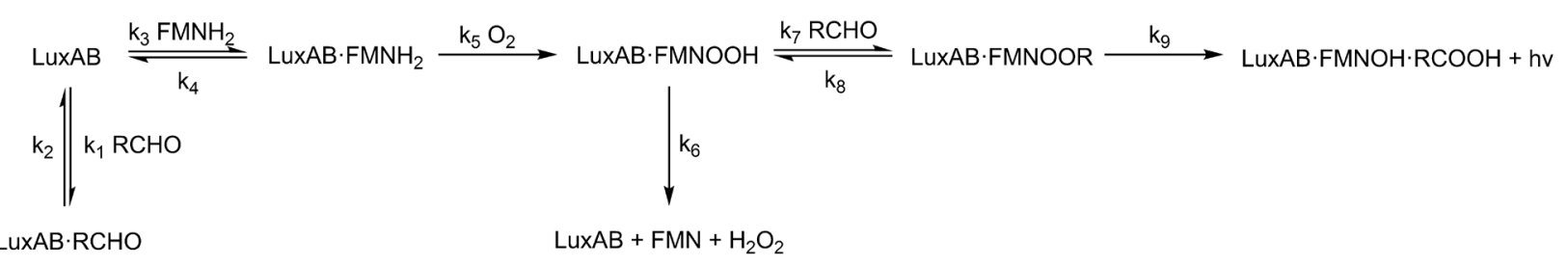

Figure 1. Reaction scheme of the bacterial bioluminescence reaction. LuXAB: luciferase, RCHO: fatty aldehyde, $\mathrm{RCOOH}$ : fatty acid, $\mathrm{FMN}$ : flavin mononucleotide, $\mathrm{FMNH}_{2}$ : reduced $\mathrm{FMN}, \mathrm{O}_{2}$ : molecular oxygen, $\mathrm{H}_{2} \mathrm{O}_{2}$ : hydrogen peroxide, $\mathrm{FMNOOH}$ : C4a-peroxy-FMN, FMNOOR: FMN-C4aperoxyhemiacetal, FMNOH: C4a-hydroxy-FMN. 
A

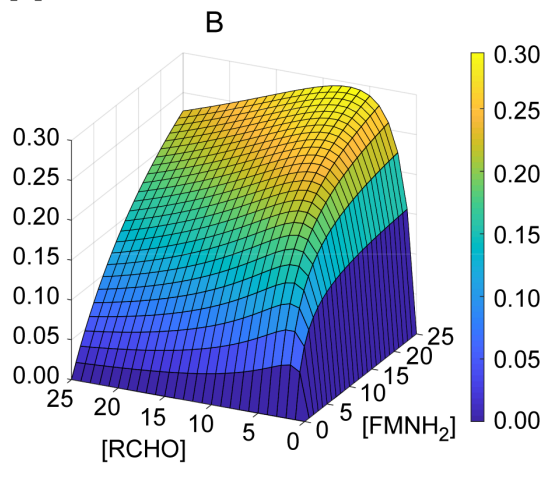

B

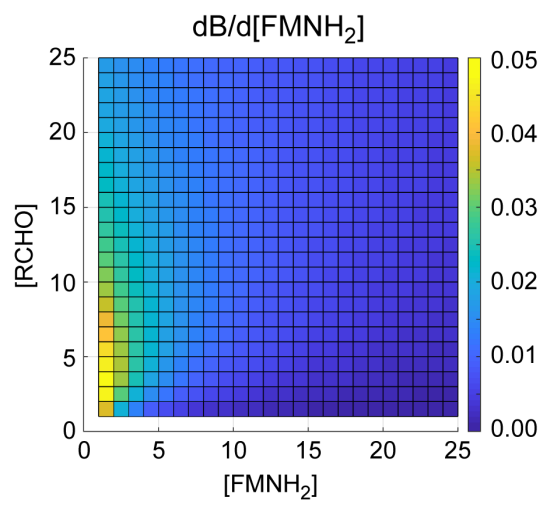

D

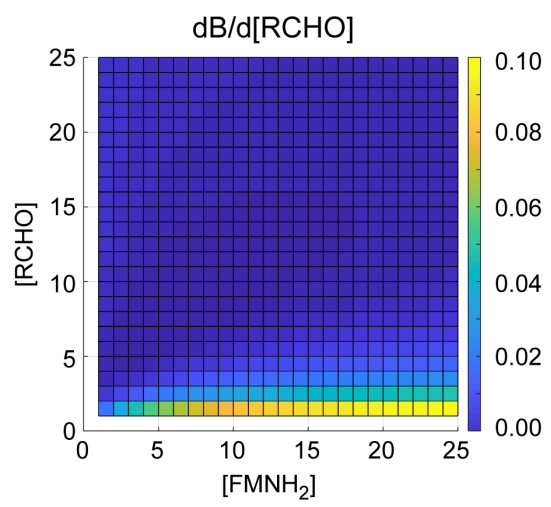

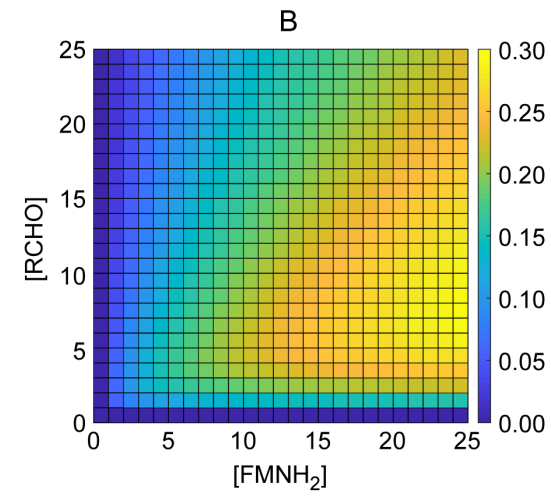

C

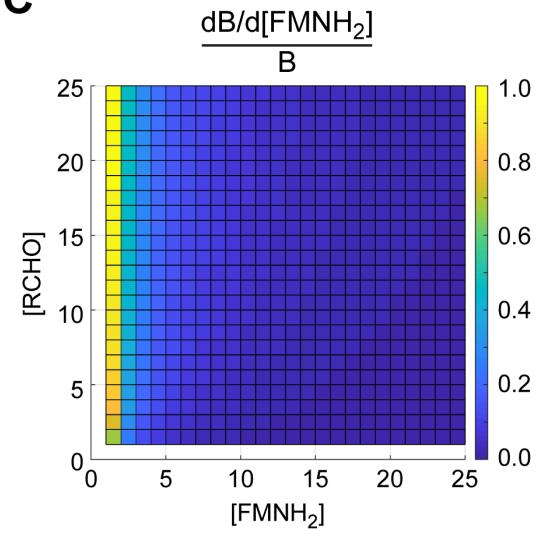

E

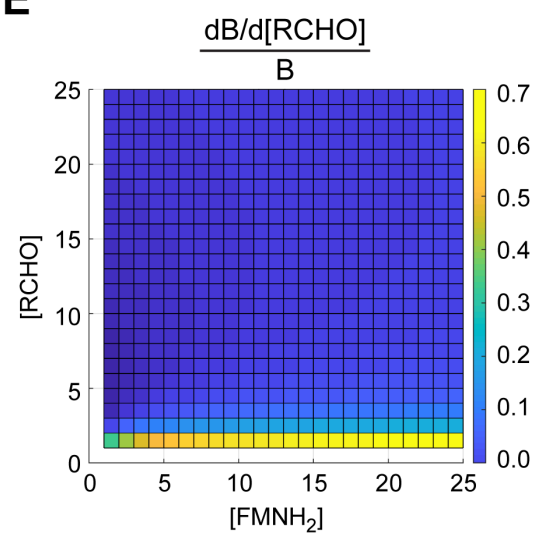

Figure 2. Qualitative model of the bioluminescence signal. (A) Bioluminescence signal B as a function of the concentrations of the substrates $\mathrm{RCHO}$ (aldehyde) and $\mathrm{FMNH}_{2}$. B was calculated based on the reaction scheme in Fig. 1 by setting all rate constants and the concentrations of luciferase and oxygen to 1. Left: side view, right: top view. (B) Derivative of the bioluminescence signal $\mathrm{B}$ with respect to the concentration of $\mathrm{FMNH}_{2}$, plotted as a function of the substrate concentrations. (C) Derivative of $B$ with respect to the concentration of $\mathrm{FMNH}_{2}$, normalized to $\mathrm{B}$. (D) Derivative of $B$ with respect to the concentration of $\mathrm{RCHO}$. (E) Derivative of $\mathrm{B}$ with respect to 
the concentration of $\mathrm{RCHO}$, normalized to $\mathrm{B}$. High values in $(\mathrm{C})$ and $(\mathrm{E})$ represent large relative changes of the bioluminescence signal with the respective substrate concentration and therefore optimal conditions for screening. Values are represented by their color as indicated in the colorbar in arbitrary units. 

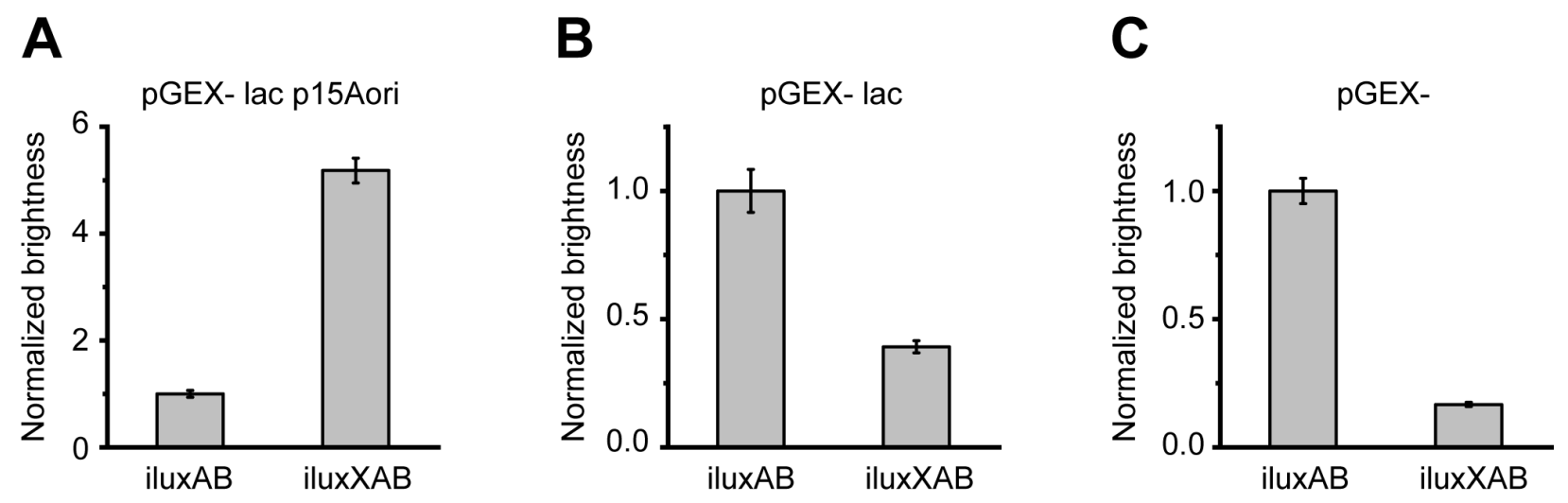

Figure 3. Mutagenesis and screening of iluXAB. Plots show the relative bioluminescence signal normalized to iluxAB. (A) For mutagenesis of ilux $A B$, ilux $A B$ was expressed at low levels from the vector pGEX(-) lac $\mathrm{p} 15$ Aori whereas iluxCDEfrp was simultaneously expressed at high levels from pGEX(-) Kan. Repeated rounds of mutagenesis resulted in the brighter variant iluXXAB. (B) Relative brightness of iluxAB and iluxXAB expressed from pGEX(-) lac. iluxCDEfrp was expressed from the same vector. (C) Relative brightness of ilux $A B$ and ilux $X A B$ expressed from pGEX(-). iluxCDEfrp was expressed from the same vector. Error bars represent SD of 5 different clones. 

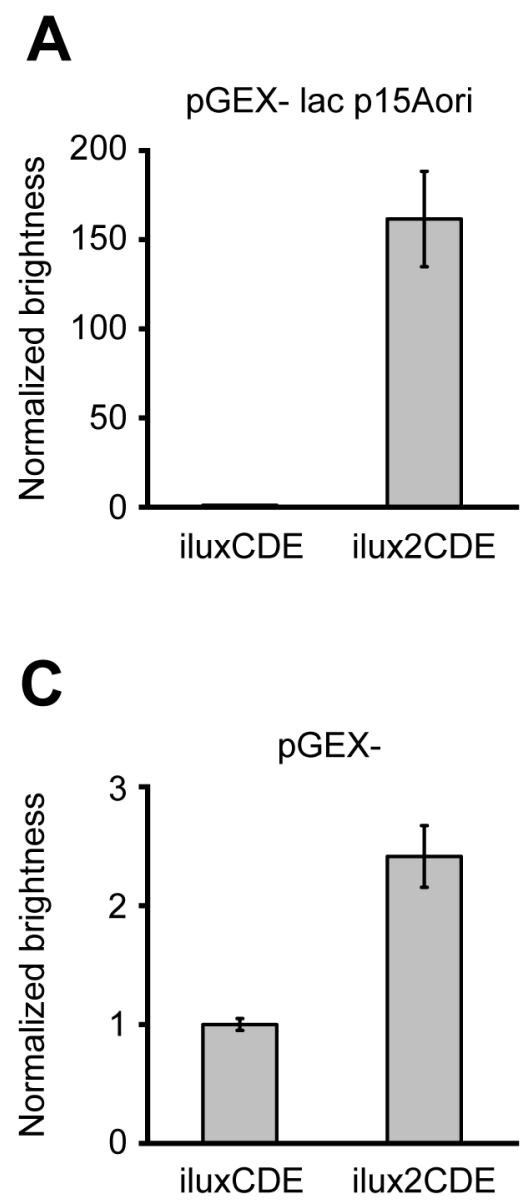

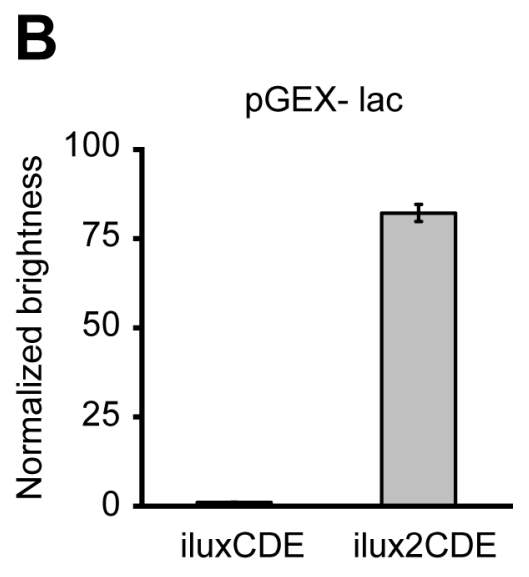

D

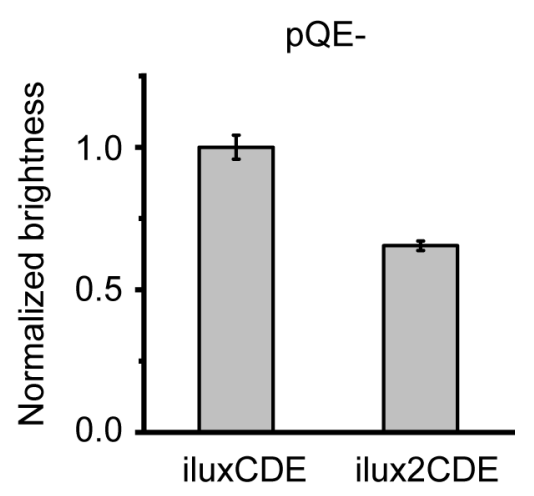

Figure 4. Mutagenesis and screening of iluxCDE. Plots show the relative bioluminescence signal normalized to iluxCDE. (A) For mutagenesis of iluxCDE, iluxCDE was expressed at low levels from the vector pGEX(-) lac p15Aori whereas iluxABfrp was simultaneously expressed at high levels from pGEX(-) Kan. Repeated rounds of mutagenesis resulted in the brighter variant ilux2CDE. (B) Relative brightness of iluxCDE and ilux2CDE expressed from pGEX(-) lac. iluxABfrp was expressed from the same vector. (C) Relative brightness of iluxCDE and ilux2CDE expressed from pGEX(-). iluxABfrp was expressed from the same vector. (D) Relative brightness of iluxCDE and ilux2CDE expressed from pQE(-). iluxABfrp was expressed from the same vector. Error bars represent SD of 5 different clones. 
A

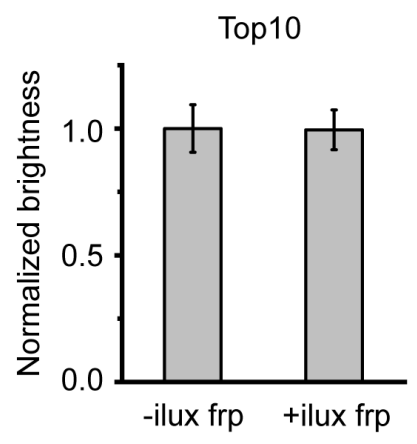

B

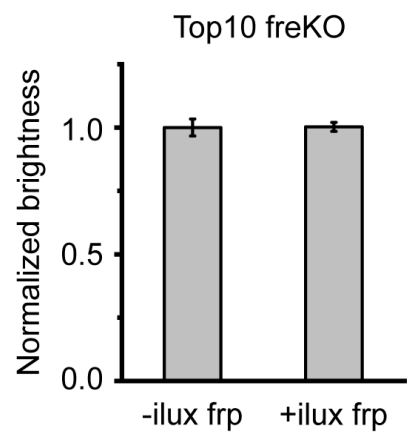

C

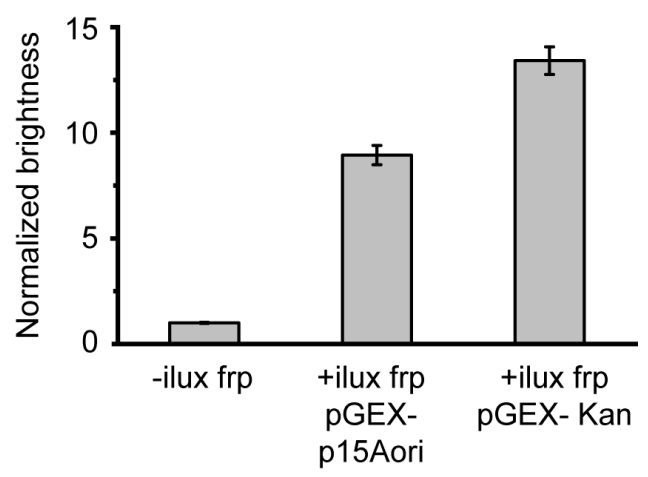

Figure 5. Mutagenesis and screening of ilux frp. Plots show the relative bioluminescence signal normalized to iluxCDABE without frp. (A) Relative brightness of iluxCDABE expressed from pGEX(-) Kan with the empty vector pGEX(-) lac p15Aori (left) and with additional expression of ilux frp from pGEX(-) lac p15Aori (right) in Top10 cells. (B) Relative brightness of iluxCDABE expressed from pGEX(-) Kan with the empty vector pGEX(-) lac p15Aori (left) and with additional expression of ilux frp from pGEX(-) lac p15Aori (right) in a Top10 fre knockout (freKO) strain. (C) Relative brightness of iluxCDABE pGEX(-) Kan in the Top10 freKO strain without additional expression of ilux frp (i.e., with the empty vector pGEX(-) p15Aori) (left), with additional expression of ilux frp from pGEX(-) p15Aori (middle) and with additional expression of ilux frp from the same vector pGEX(-) Kan (right). For mutagenesis of ilux frp, expression from pGEX(-) p15Aori (together with iluxCDABE from pGEX(-) Kan) was chosen due to the sufficiently increased brightness compared to cellular $\mathrm{FMNH}_{2}$ production only. Error bars represent SD of 5 different clones. 


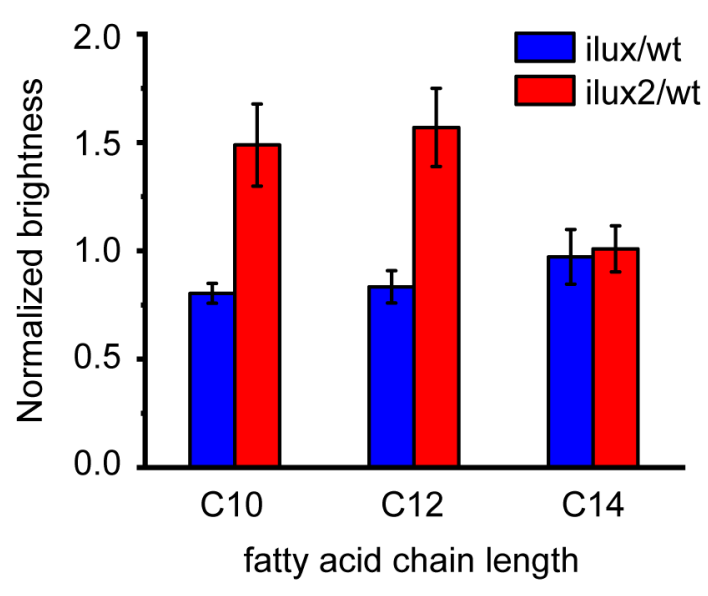

Figure 6. Comparison of aldehyde production of LuxCDE wt, iLuxCDE and iLux2CDE from saturated fatty acids of different chain length. Top10 cells expressing luxCDE wt, iluxCDE or ilux2CDE from pGEX(-) Kan were incubated in LB medium containing $300 \mu \mathrm{M}$ decanoic, dodecanoic or tetradecanoic acid for $5 \mathrm{~min}$. Subsequently, Top10 cells expressing iluxABfrp from pGEX(-) Kan were added to detect the aldehyde produced by LuxCDE by bioluminescence. Imaging was performed at room temperature. For each fatty acid, the bioluminescence signal was normalized to LuxCDE wt. Error bars represent SD of 4 different clones. 


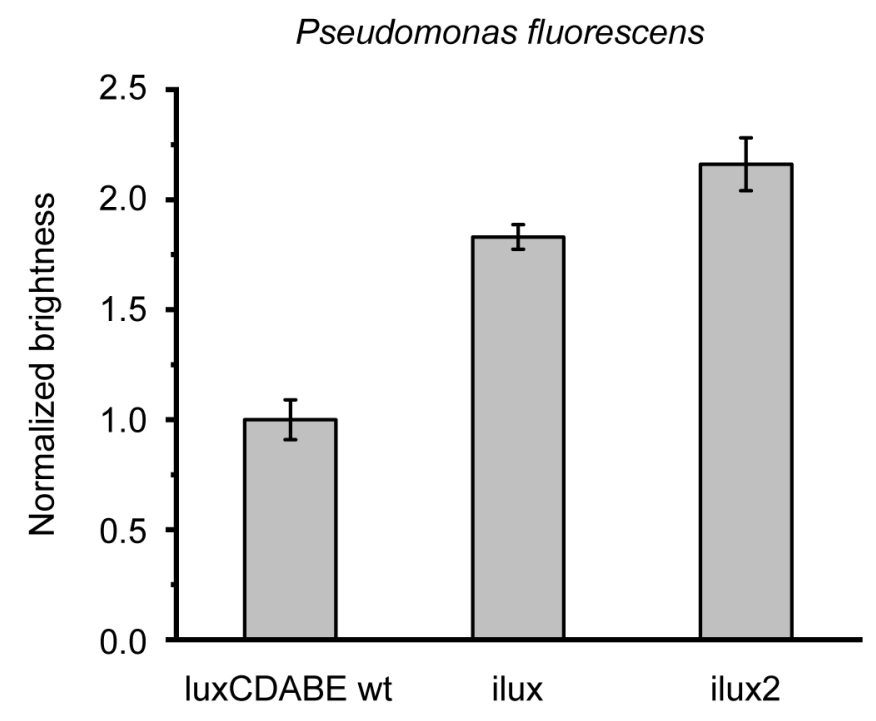

Figure 7. Comparison of luxCDABE wt, ilux and ilux2 in Pseudomonas fluorescens. The indicated lux operons were expressed in P. fluorescens from the vector pJOE7771.1 at $30^{\circ} \mathrm{C}$. The bioluminescence signal was normalized to the luxCDABE wt operon. Error bars represent SD of 5 different clones. 


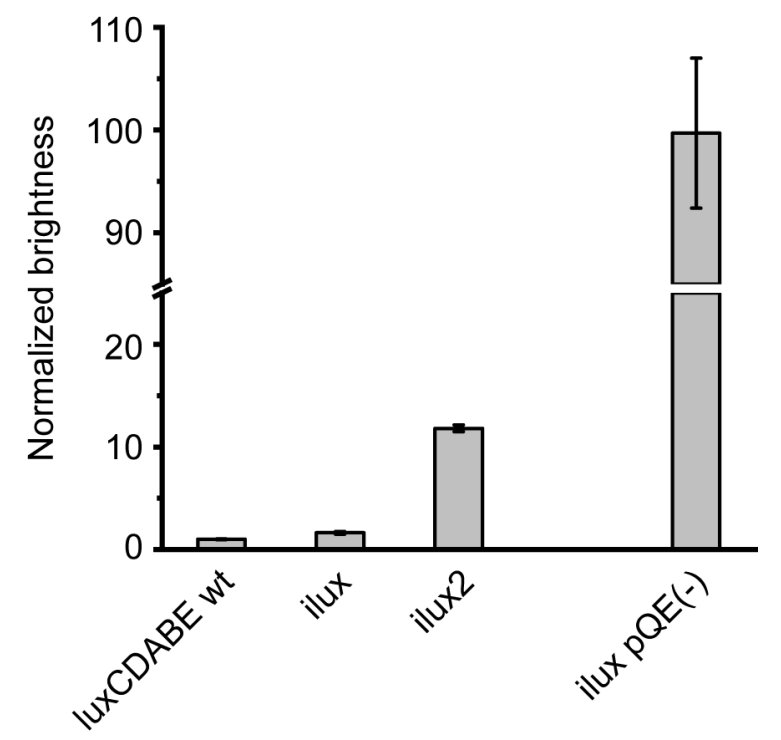

Figure 8. Comparison of bioluminescence emission from the chromosomally expressed operons luxCDABE wt, ilux and ilux2 (left) with ilux expressed from the vector pQE(-) (right) in Top10 cells. The bioluminescence signal was normalized to the luxCDABE wt strain. Error bars represent SD of 5 different clones. 
A

luxCDABE wt

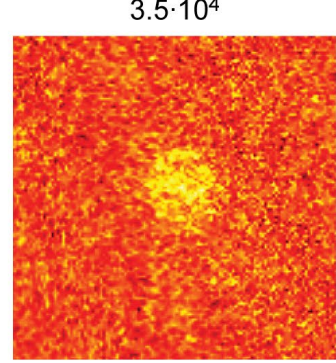

B

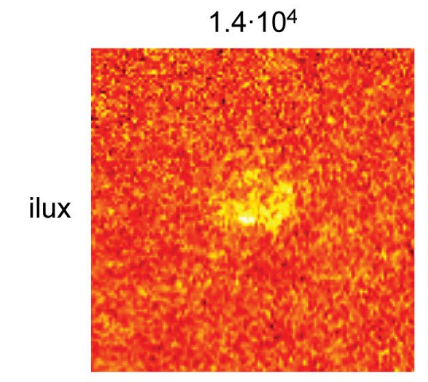

C

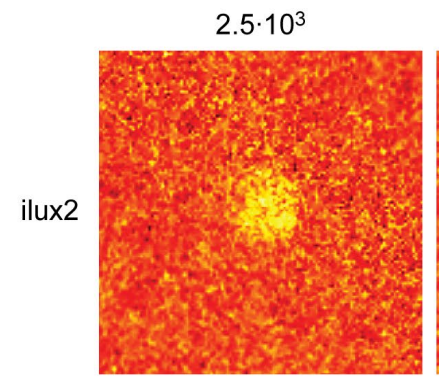

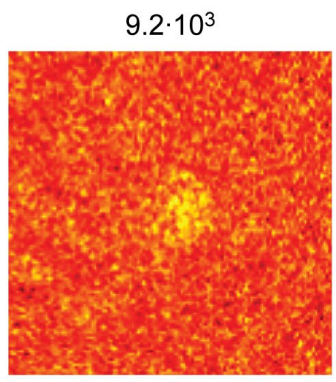

$2.3 \cdot 10^{4}$

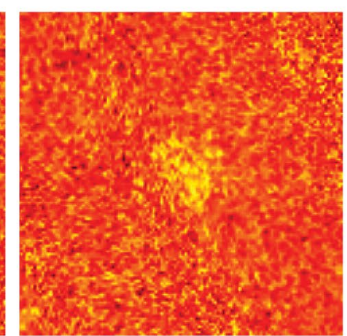

$9.2 \cdot 10^{3}$

$1.6 \cdot 10^{3}$

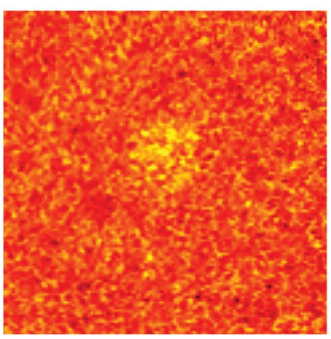

$1.5 \cdot 10^{4}$

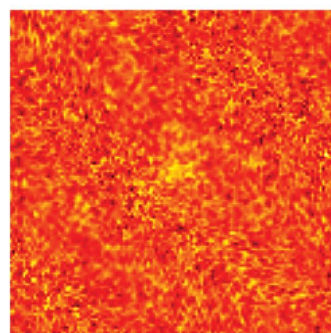

$6.9 \cdot 10^{3}$

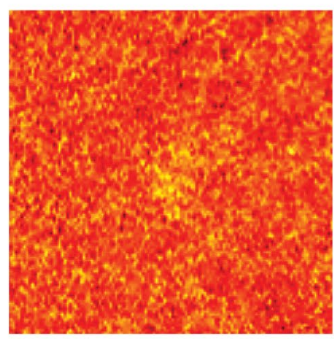

$1.2 \cdot 10^{3}$

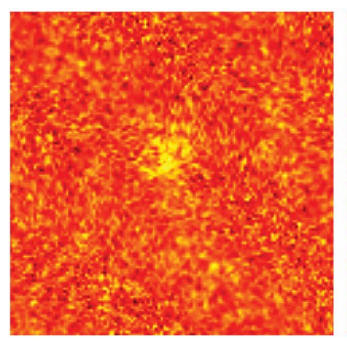

$1.0 \cdot 10^{4}$

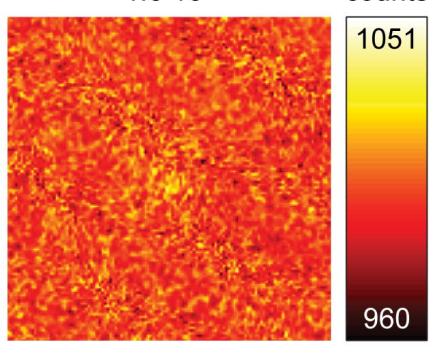

$4.6 \cdot 10^{3}$

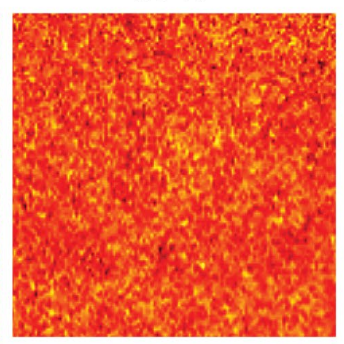

counts

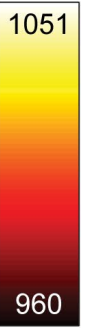

960

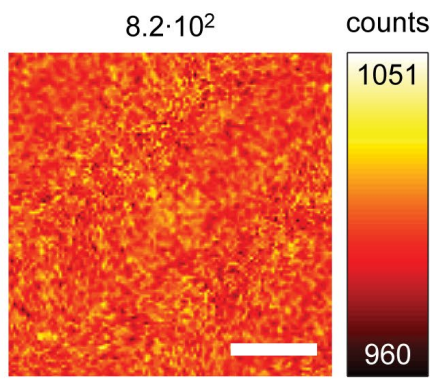

D

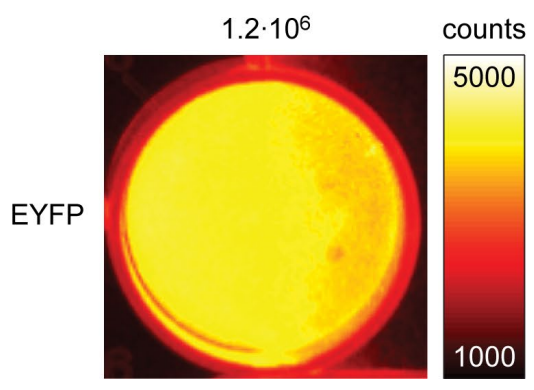

Figure 9. Detection of Top10 strains chromosomally labeled with (A) luxCDABE wt, (B) ilux, (C) ilux2 or (D) EYFP on mashed potatoes. The indicated numbers of colony-forming units were applied in $1 \mu \mathrm{l}$ PBS onto mashed potatoes in 24-well plates. Imaging was performed at room temperature. Bioluminescence from the luxCDABE wt, ilux and ilux2 strains was imaged with an exposure time of $10 \mathrm{~min}$. Fluorescence of the EYFP strain was excited at $520 \mathrm{~nm}$ and imaged with an exposure time of $1 \mathrm{~s}$. Scalebar: $5 \mathrm{~mm}$. 

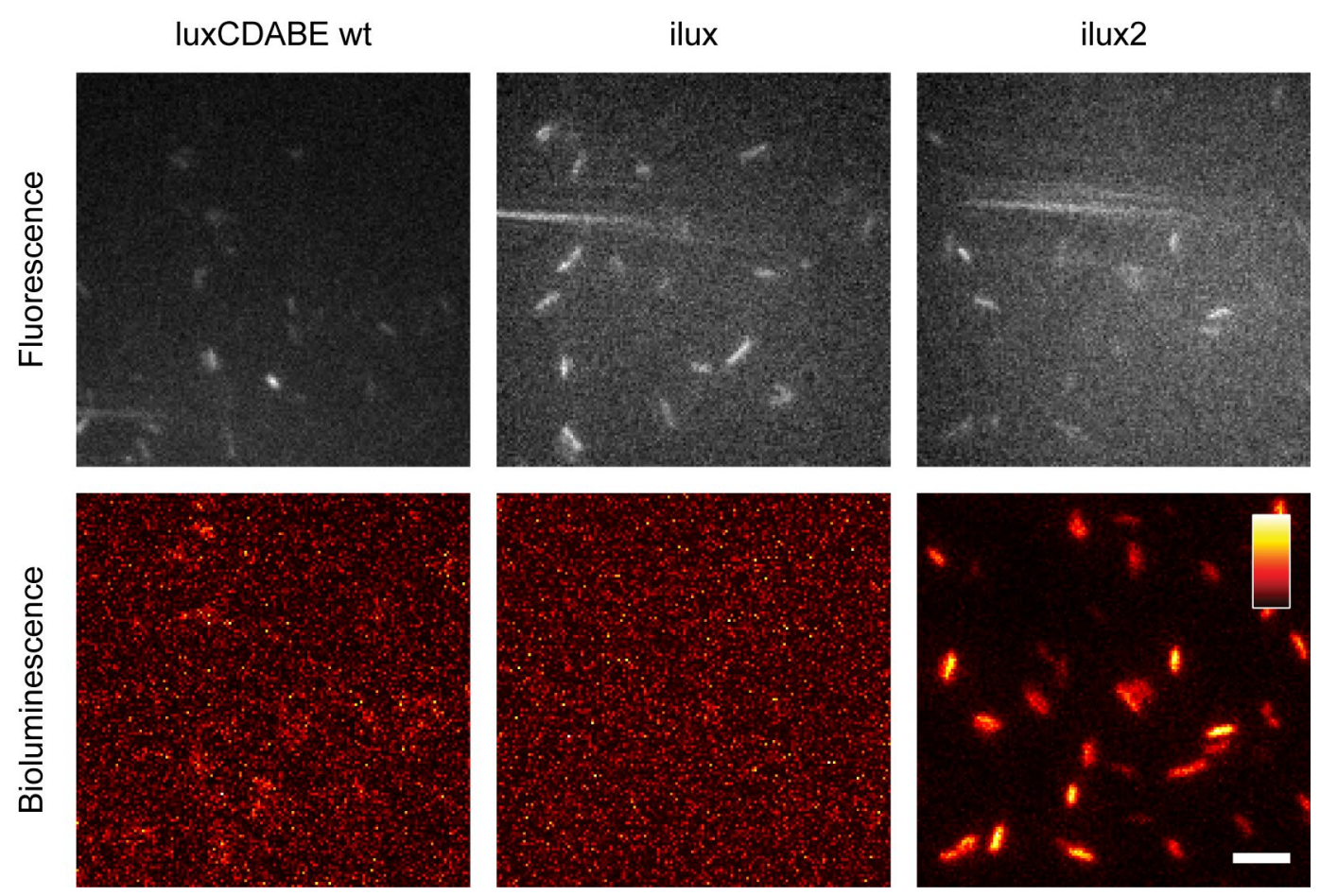

Figure 10. Imaging of single Top10 cells with luxCDABE wt, ilux or ilux2 inserted into the chromosome. All three strains were transformed with EYFP pGEX(-) to detect the cells by their fluorescence excited at $491 \mathrm{~nm}$ (top row). Bioluminescence images were taken with an exposure time of $10 \mathrm{~min}$. Imaging was performed at $37^{\circ} \mathrm{C}$. The colormap of each image was scaled to the minimum and maximum pixel values. Scalebar: $5 \mu \mathrm{m}$. 
A

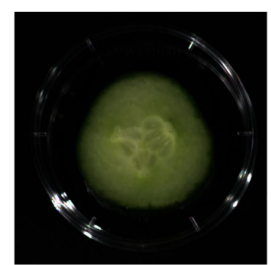

B

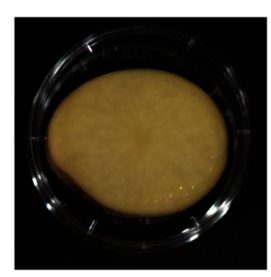

C

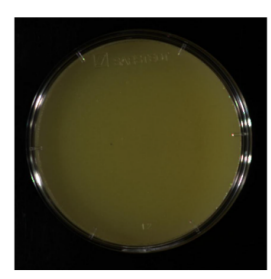

D

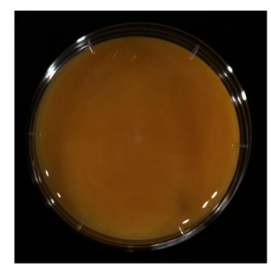

E

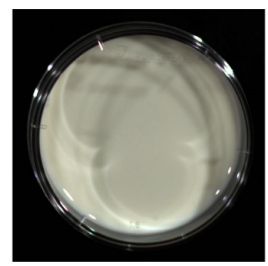

$\mathrm{Oh}$

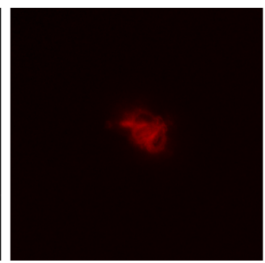

$\mathrm{Oh}$

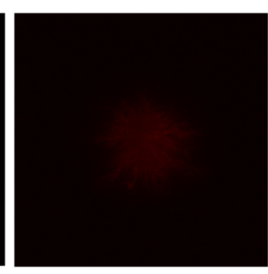

$0 \mathrm{~h}$

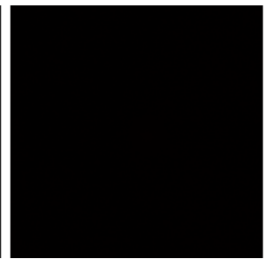

$\mathrm{Oh}$

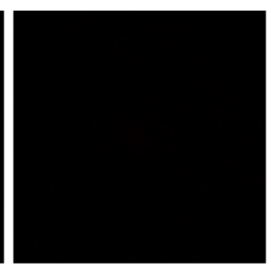

$\mathrm{Oh}$

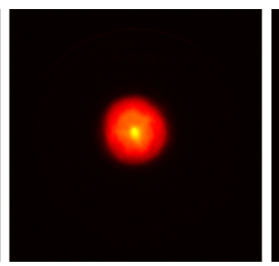

$6 \mathrm{~h}$

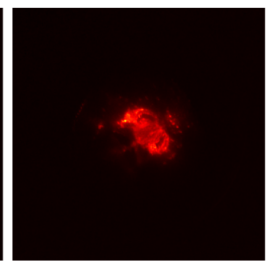

$6 \mathrm{~h}$

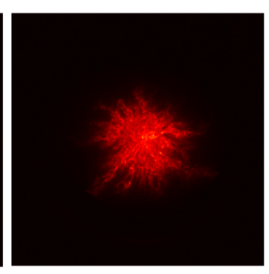

$6 \mathrm{~h}$

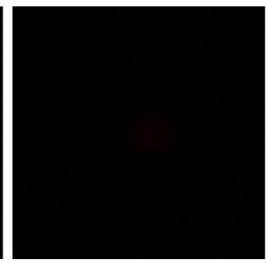

$6 \mathrm{~h}$

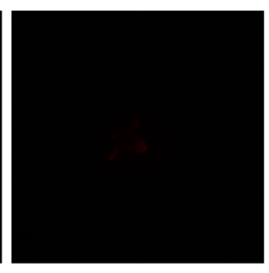

$6 \mathrm{~h}$

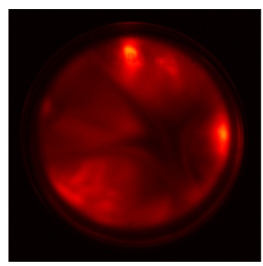

$12 \mathrm{~h}$

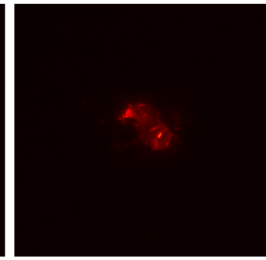

$12 \mathrm{~h}$

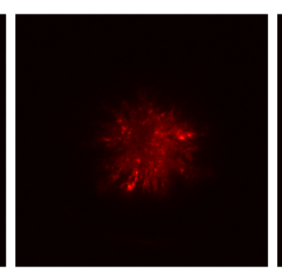

$12 \mathrm{~h}$

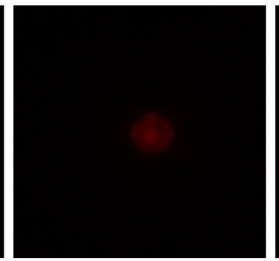

$12 \mathrm{~h}$

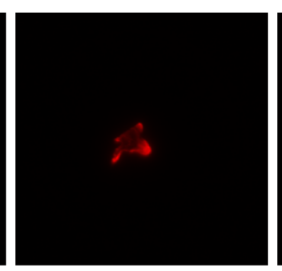

$12 \mathrm{~h}$

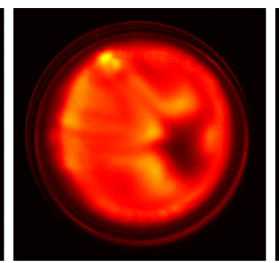

$18 \mathrm{~h}$

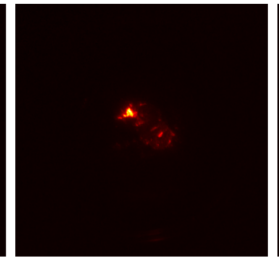

$18 \mathrm{~h}$

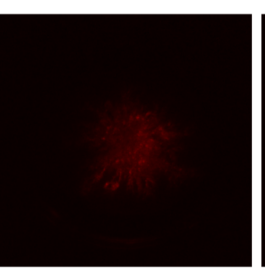

$18 \mathrm{~h}$

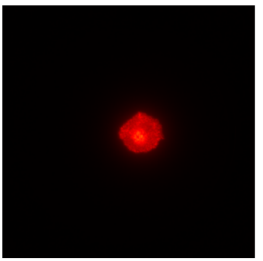

$18 \mathrm{~h}$

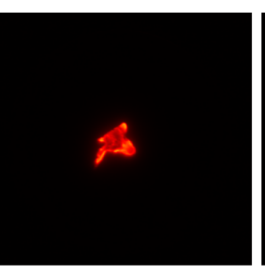

$18 \mathrm{~h}$

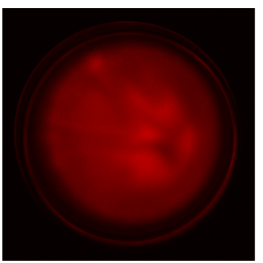

$24 \mathrm{~h}$

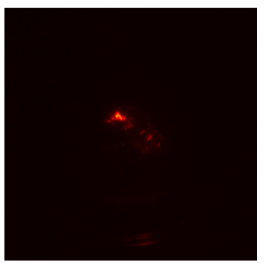

$24 \mathrm{~h}$

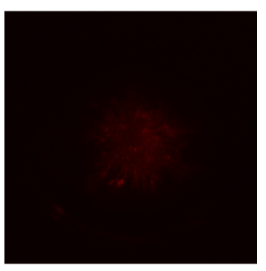

$24 \mathrm{~h}$

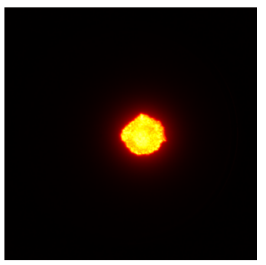

$24 \mathrm{~h}$

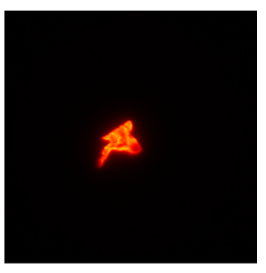

$24 \mathrm{~h}$

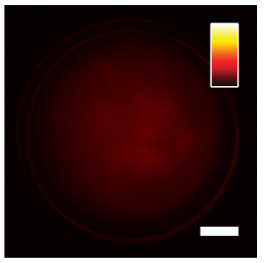

Figure 11. Growth of $E$. coli Top10 cells on different food products. Food samples were placed in $6 \mathrm{~cm}$ dishes and inoculated with Top10 cells chromosomally expressing ilux2. Bioluminescence images were taken at room temperature with an Amersham Imager AI 600 RGB after the indicated time periods. The colormap of each time series was scaled to the minimum and maximum pixel values. The following food products and exposure times per bioluminescence image were used: (A) cucumber, $1 \mathrm{~min},(B)$ raw potato, $1 \mathrm{~min}$, (C) mashed potatoes, $5 \mathrm{~s}$, (D) egg yolk, $5 \mathrm{~s}$, (E) milk, $1 \mathrm{~min}$. Complete time series are shown in Movies 1-5. Scalebar: $1 \mathrm{~cm}$. 


\section{Movies}

\section{Movie 1}

E. coli Top10 strain chromosomally expressing ilux2 grown on cucumber. A slice of cucumber was inoculated with $1 \cdot 10^{6}$ colony-forming units (cfu). Bioluminescence images were taken at room temperature with an Amersham Imager Al 600 RGB using an exposure time of 1 min per image.

\section{Movie 2}

E. coli Top10 strain chromosomally expressing ilux2 grown on potato. A slice of raw potato was inoculated with $1 \cdot 10^{6} \mathrm{cfu}$. Bioluminescence images were taken at room temperature with an Amersham Imager Al 600 RGB using an exposure time of 1 min per image.

\section{Movie 3}

E. coli Top10 strain chromosomally expressing ilux2 grown on mashed potatoes. A dish filled with mashed potatoes was inoculated with $5 \cdot 10^{5} \mathrm{cfu}$. Bioluminescence images were taken at room temperature with an Amersham Imager Al 600 RGB using an exposure time of $5 \mathrm{~s}$ per image.

\section{Movie 4}

E. coli Top10 strain chromosomally expressing ilux2 grown on egg yolk. A dish filled with raw egg yolk was inoculated with $5 \cdot 10^{5} \mathrm{cfu}$. Bioluminescence images were taken at room temperature with an Amersham Imager Al 600 RGB using an exposure time of 5 s per image.

\section{Movie 5}

E. coli Top10 strain chromosomally expressing ilux2 grown in milk. A dish filled with UHT milk was inoculated with $3 \cdot 10^{6} \mathrm{cfu}$. Bioluminescence images were taken at room temperature with an Amersham Imager Al 600 RGB using an exposure time of 1 min per image.

\section{Additional Files}

\section{Supplementary file 1}

Nucleotide sequences of the ilux2 genes. The sequences of ilux $2 A$, ilux $2 B$ and ilux $2 f r p$ are identical to iluxA, iluxB and iluxfrp, respectively. The sequence of ilux2E is the same as in the wild-type lux operon. 